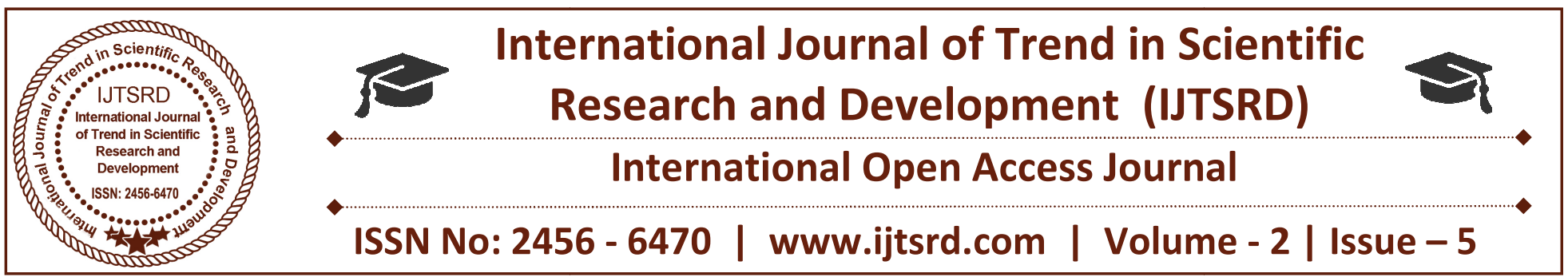

\title{
SVM Classifiers at it Bests in Brain Tumor Detection using MR Images
}

\author{
Dr. R Manjunatha Prasad ${ }^{1}$, Roopa B S ${ }^{2}$ \\ ${ }^{1}$ Professor \& Head, Department of Electronics and Communication, DSATM, Bangalore, Karnataka, India \\ ${ }^{2}$ Associate Professor, Department of Electronics and Communication, DBIT, Bangalore, Karnataka, India
}

\section{ABSTRACT}

This paper presents some case study frameworks to limelight SVM classifiers as most efficient one compared to existing classifiers like Otsu, k-means and fuzzy c-means. In general, Computed Tomography (CT) and Magnetic Resonance Imaging (MR) are more dominant imaging technique for any brain lesions detection like brain tumor, Alzheimer's disease and so on. MR imaging takes a lead technically for imaging medical images due to its possession of large spatial resolution and provides better contrast for the soft tissues like white matter (WM), gray matter (GM) and cerebrospinal fluid (CSF). The usual method used for classification of lesions in brain images consists of pre-processing, feature extraction, feature reduction and classification. Early detection of the tumor region without much time lapse in computation can be achieved by using efficient SVM classifier model. Brain tumor grade classifications with the assistance of morphologically selected features are extracted and tumor classification is attained using SVM classifier. The assessment of SVM classifications are evaluated through metrics termed as sensitivity, exactness and accuracy of segmentation. These measures are then compared with existing methods to exhibit the SVM classifier as significant classifier model.

KEYWORD: Computed Tomography(CT),Magnetic Resonance(MR), white matter(WM),gray matter(GM) ,cerebrospinal fluid(CSF),Support vector machine(SVM).

\section{INTRODUCTION}

In India, every year about 40,000 to 50,000 persons are diagnosed with brain tumor. Of these $20 \%$ are children. $35 \%$ men and $36 \%$ women in 5 years survey. During the period of treatment, this grade measurement gives the information about the rate of growth of tumor .Medical image processing specially MRI imaging modalities is the most challenging and innovative field. MRI is a 3-D non-invasive imaging modality, which is best suited for soft tissue abnormality detection. Classification is a data mining technique that helps in mapping any nonlinear data into a well structured data to help the analyzer to extract or discover the information from the subject classified. It provides decision making intellectually. Classifiers are of two types: supervised classifiers and unsupervised classifiers. Supervised classifiers adopt two steps, firstly it learns the data and secondly based on the learning the algorithm is devised.

In supervised classifiers, correct targets of the subjects are known and are assigned as input during first process of learning. This method is usually fast and accurate. The main aim of the supervised classifier like SVMs is to analyze a large data and realistic descriptions of it are modelled using the feature extracted from the given subject. For linear or structured data the linear SVMs are used for classification, but in realistic the available data are unstructured or non linear hence the SVMs with specific kernels are used to handle specific problem.

SVMs are at its best even when the data are large and complex. The important attribute of SVM kernels are, it can be applied directly to data without inclusion of feature extraction. SVMs deterministic speed and memory usage are good. SVMs have the ability to learn high dimensional data. SVMs have applications 
like Handwriting recognition, text categorization and image classification.

Addressing decision boundary:

$\bar{w}$ is a weight vector and $\mathrm{b}$ is a offset.

$\mathrm{C}$ is a constant

$\bar{w} \cdot \bar{u} \geq c$

$\bar{w} \cdot \bar{u}+b \geq 0$ If that's true then + samples

Equation (2) is a Decision Rule.

If $\mathrm{c}=-\mathrm{b}$

$\bar{w} \cdot \overline{x_{+}}+b \geq 1$

$\bar{w} \cdot \overline{x_{-}}+b \geq-1$

Let $y_{i}$ be such that $y_{i}=+1$ for + ve samples (5)

$$
\begin{aligned}
& y_{i}=-1 \text { for }- \text { ve samples } \\
& \text { Then } y_{i}\left(\overline{x_{i}} \cdot \bar{w}+b\right) \geq 1 \\
& y_{i}\left(\overline{x_{i}} \cdot \bar{w}+b\right)-1 \geq 0 \\
& y_{i}\left(\overline{x_{i}} \cdot \bar{w}+b\right)-1=0
\end{aligned}
$$

Equation (9) is the constrained optimization, and then the Lagrange multiplier is given by

$$
\sum \alpha_{i} y_{i} \bar{x}_{i} \cdot \bar{u}+b \geq 0
$$

Equation (10) is the Decision rule which depends on dot product called feature space.

Some of the SVM Kernels are

1. $\mathrm{K}$ SVMs where $\mathrm{K}$ provides dot product $k(x, y)=(\bar{x} \cdot \bar{y}+1)^{d}$

2. Radial Basis Style kernel function RSVM $k(x, y)=\exp \left(-\|x-y\|^{2} \div 2 \sigma^{2}\right)$

3. Neural-net style kernel $k(x, y)=\tanh (k x . y-\delta)$

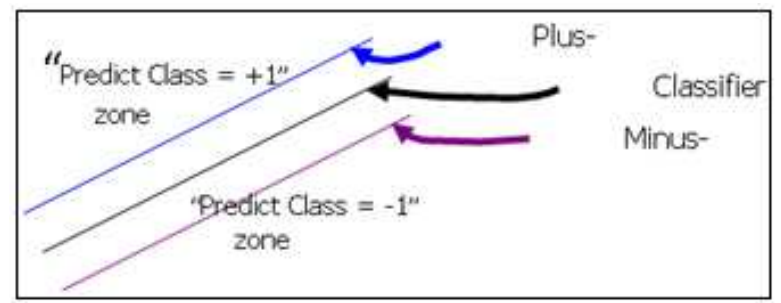

Fig.1. Maximum margin of SVM

Performance measure of SVM classifiers are

$\%$ sensitivity $=\mathrm{TP} /(\mathrm{TP}+\mathrm{FN}) * 100$

$\%$ Exactness $=\mathrm{TN} /(\mathrm{TN}+\mathrm{FP}) * 100$

$\%$ Accuracy $=(\mathrm{TP}+\mathrm{TN}) /(\mathrm{TP}+\mathrm{TN}+\mathrm{FP}+\mathrm{FN}) * 100$
Where True Positive (TP) is Abnormal correctly detected as abnormal

True Negative (TN) is Normal correctly detected as normal

False Positive (FP) is Normal incorrectly detected as abnormal

False Negative (FN) is abnormal incorrectly detected as normal

\section{LITERATURE SURVEY}

Brain Tumor has been one of the alarming issue of increasing mortality rate in the world. To detect tumor at the earliest would be the corrective measure for which MR Imaging is more apt for deriving the details of the brain for acquiring significant information.

Pre-processing of the image is performed using normalized histogram to filter out the undesired signal or noise from it. The second step after pre-processing technique is various textures based and intensity features are extracted. After extraction of the features, the feature reduction is carried out by principal component analysis (PCA) [2]. Finally the support vector machines (SVM) is used to classify and measure performance metrics like index, overlap fractions and extra fractions. The noise is reduced using Gaussian filter. In feature extraction, the texture features such as skewness (which measures asymmetricity of the data around the mean), coarseness (based on its shape) and standard deviation (to depict variations that exist from the expected value or mean) are assessed to identify the abnormal cells for tumor detection. K-NN algorithm and linear SVM are used for classification. In this paper the classification efficiency is attained about $94 \%$ in case of linear SVM compared to $92 \%$ in case of K-NN.

function Garima singh et. al., [4] used normalized histogram and k-mean segmentation. Here Naive Bayes classifies and the support vector machines are used for classification. In this unsupervised $\mathrm{k}$ - means segmentation is adopted. The centres of $\mathrm{K}$ clusters are initialized. Euclidean distances between the cluster centroids are computed. Depending on the Euclidean measure the pixel with closest centre is assigned for cluster. The cluster centre is recomputed. This process is continued until it confines to one solution. Cluster pixels are then clustered into an image. In this paper Bayes classifier has $87.23 \%$ of classifying efficiency. SVM classifier is $91.49 \%$ efficient classifier. 
Bias correction [5] is performed on MR images by applying Hidden Markov Random Field algorithm. The tumor present is asymmetrical in nature hence the intensity is also higher. Thus method includes separation of the brain in symmetric slices and then comparing them voxel based by subtraction method. To extract the abnormal cell region, region growing technique is implied with voxel having highest intensity as seed point. Tumor shapes indicate different stages or grades of tumor. SVM with linear kernel exhibits much better performance compared to other classifiers. The statistical texture and morphological features selected is fed to different classifiers. The maximum accuracy of classification is achieved from SVM.

Medical image segmentation is highly influenced by noise and the noise can be declined using low pass filter but low pass blurs the image and degrades edges. To avoid this degrading further median filter is adopted which takes care of sharp edges but fine details are lost. Thus an isotropic filter is implemented which provide goodness of low pass filter and median filter hence overcoming the drawbacks, Active contour model(ACM) [6] is one of the efficient segmentation algorithm where by growing curve, the intended object can be separated out. Discrete wavelet transform (DWT) is used for feature derivation, but DWT increases the power as feature vector is tried to reduce, Independent component analysis (ICA) is used to reduce the vector size of the feature. The author believes supervised methods of classification are better in accuracy than the unsupervised method, as SVMs can handle complex data or high dimensional data. In this work nonlinear kernels of SVMs are used like linear, polynomial, quadratic and RBF etc. The result in this paper concludes that nonlinear SVMs especially exponential form expands the width between samples to the extent where other kernels cannot achieve. RBFK renders best classification accuracy compared to other nonlinear kernels SVMs.

\section{PROPOSED METHODOLOGY}

SVM is more efficient as only a support vectors play a vital role in deciding the decision boundary for classification hence the memory usage is efficiently used with the help of kernels, SVMs can map feature space into a high dimension so as to represent as collection of linearly independent set. Kernel trick is employed based on the need to build linear model over high dimension space.
The state-of-the-art algorithm is sequential minimal optimizations which executed quadratic form and are widely used.

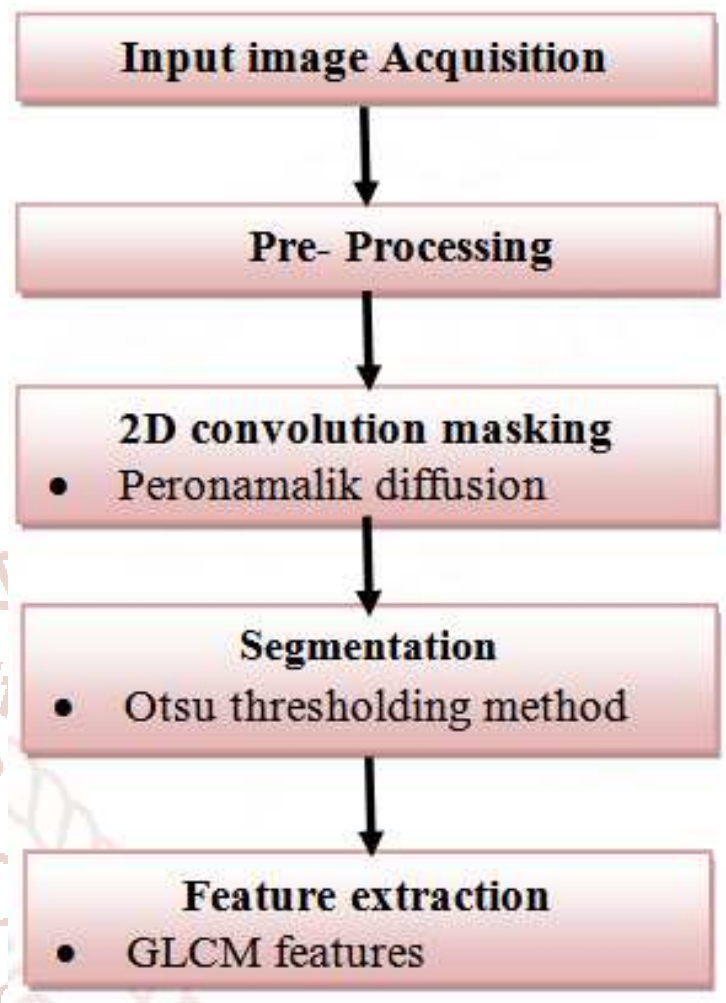

Fig.2. Flow chart of the proposed steps for feature extraction

\section{Peronamalik diffusion}

In anisotropic diffusion, it has a function to reduce noise with inhibits smoothing at the image edges called diffusion coefficient $\operatorname{cd}(\mathrm{x})$, it corresponds to the image Gradient ( $\nabla \mathrm{I})$ [7].

The basic anisotropic diffusion PDE in 1D anisotropic diffusion is:

$$
\frac{\partial I(x, t)}{\partial t}=\operatorname{div}\left[c_{d}(x, t) \nabla I(x, t)\right]
$$

$I(x, t)$ is a signal, $x$ refers to signal axes and $t$ refers to the iteration step, cd $(x, t)$ is called the diffusion coefficient [2] [7]. The function that impedes smoothing at the edges is the diffusion coefficient $\operatorname{cd}(\mathrm{x})$.

Perona-Malik proposed [2]:

$$
\begin{aligned}
& c_{d 1}(x)=\exp \left\{-\left[\frac{\nabla l(x, t)}{K}\right]^{2}\right\} \\
& c_{d_{2}}(x)=\frac{1}{1+\left[\frac{\nabla 1(x, t)}{K}\right]^{2}}
\end{aligned}
$$


where Cd1 privileges high-contrast edges over low contrast ones, $\mathrm{Cd} 2$ privileges wide regions over smaller ones, $\nabla \mathrm{I}$ is image gradient, and Kappa $(\mathrm{K})$ is referred to as the diffusion constant or the flow constant.

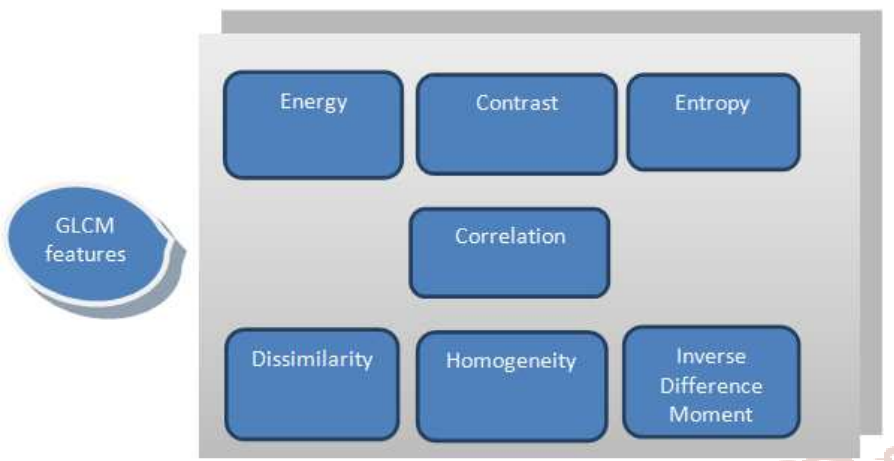

Fig.3. GLCM feature extraction

\section{Kernel Functions}

$$
K\left(\mathbf{x}_{\mathrm{i}}, \mathbf{X}_{\mathrm{j}}\right)=\left\{\begin{array}{lr}
\mathbf{X}_{\mathrm{i}} \cdot \mathbf{x}_{\mathrm{j}} & \text { Linear } \\
\left(\gamma \mathbf{X}_{\mathrm{i}} \cdot \mathbf{x}_{\mathrm{j}}+\mathrm{C}\right)^{\mathrm{d}} & \text { Polynomial } \\
\exp \left(-\gamma\left|\mathbf{X}_{\mathrm{i}}-\mathbf{X}_{\mathrm{j}}\right|^{2}\right) & \mathrm{RBF} \\
\tanh \left(\gamma \mathbf{X}_{\mathrm{i}} \cdot \mathbf{X}_{\mathrm{j}}+\mathrm{C}\right) & \text { Sigmoid }
\end{array}\right\}
$$

Where $K\left(\mathbf{x}_{\mathrm{i}}, \mathbf{X}_{\mathrm{j}}\right)=\phi\left(\mathbf{X}_{\mathrm{i}}\right) \bullet \phi\left(\mathbf{X}_{\mathrm{j}}\right)$

That is, the kernel function represents a dot product of input data points mapped into the higher dimensional feature space by transformation

\section{Gamma is an adjustable parameter of certain} kernel functions.

The RBF is by far the most popular choice of kernel types used in Support Vector Machines. This is mainly because of their localized and finite responses across the entire range of the real $\mathrm{x}$-axis.

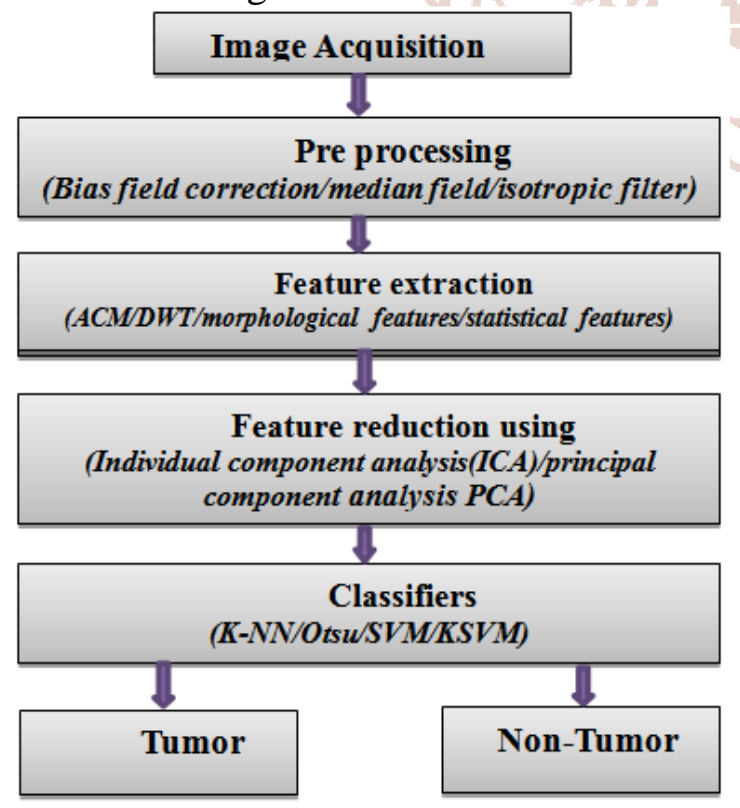

Fig.4. Flow chart of the proposed algorithm

\section{EXPERIMENTAL RESULTS}

Brain MRI image classification is an important task. In proposing work image preprocessing is done with median filtering and skull stripping method. It shows better performance. The features were extracted and SVM is used as a classifier.

Classification method KNN algorithm is one of data classification methods which has strong consistency. KNN algorithm does not show an accurate while when compared to other two classifiers.

One of the best methods for classifying any image or pattern is SVM. SVM is used to split a set of images into two various classes. The classification is done by finding the hyper-plane that differentiate the two classes.

Among the MR images collected, $18 \mathrm{MR}$ images are diseased and $56 \mathrm{MR}$ images are non-diseased or normal images. The GUI menu option opens as in Figure.
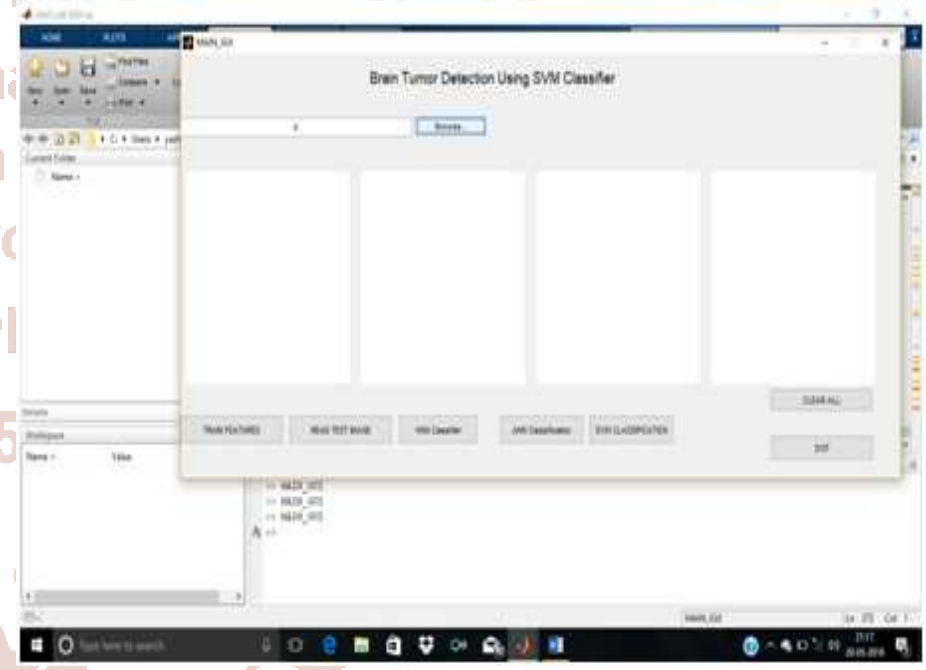

Figure.5. GUI menu

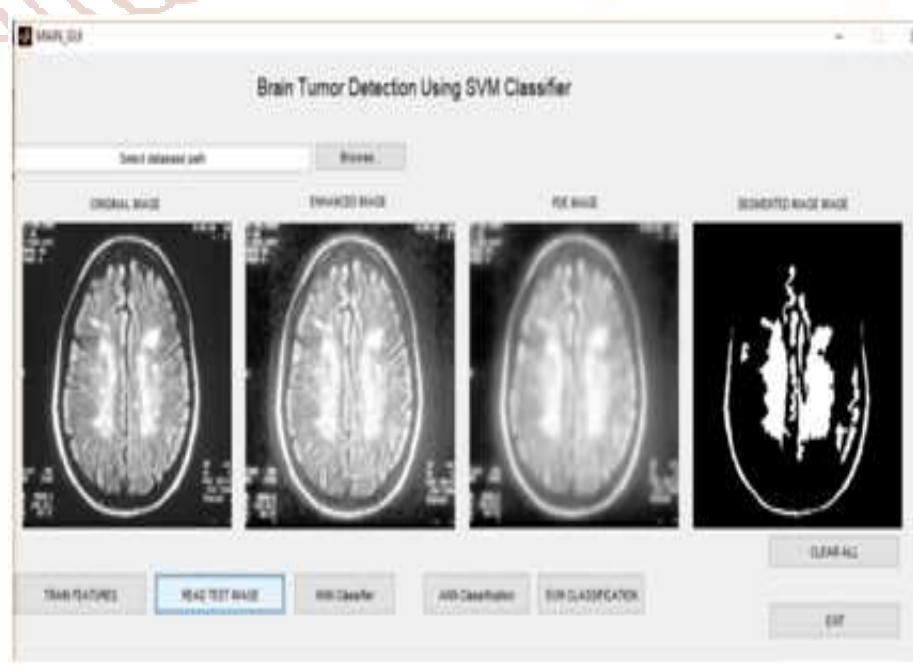

Figure.6. Image acquisition 


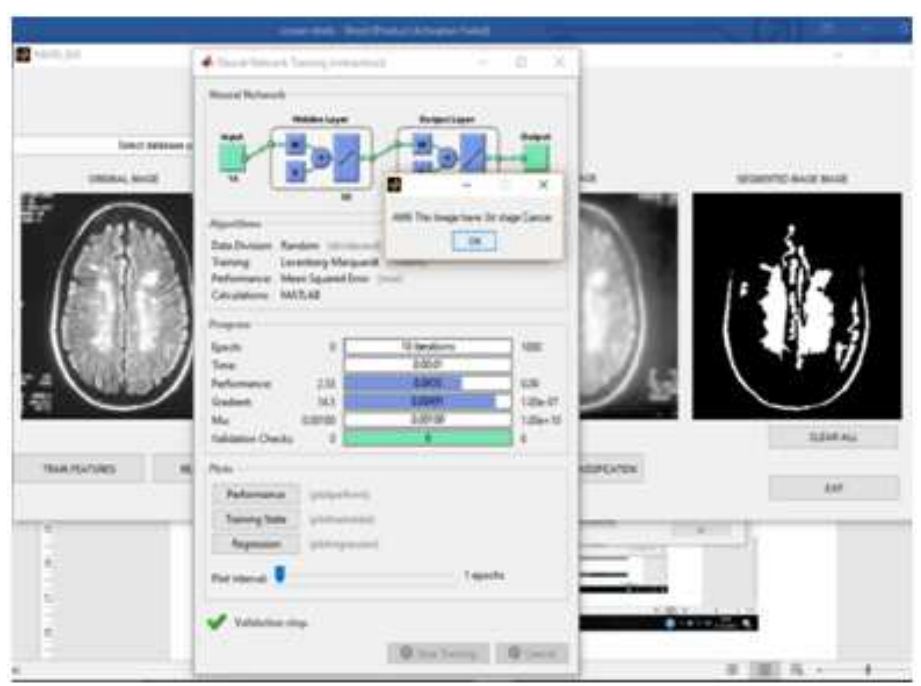

Figure.7. Neural network training

After the process of morphology and clustering, the tumor part is alone segmented and is displayed along with its properties as in Figure.7. Also, on selecting the option "RESULT", the result of the tumor either being normal, benign or malignant is displayed as in Fig.9.

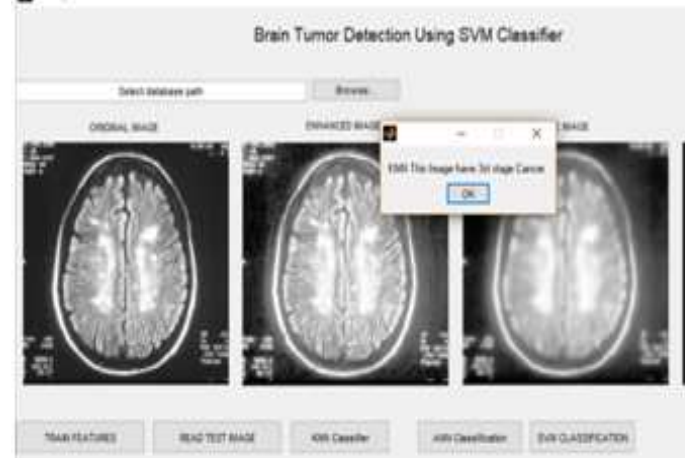

Figure.8. KNN classifier training

Classification method KNN algorithm is one of data classification methods which has strong consistency. KNN algorithm does not show an accurate while when compared to other two classifiers. Fig.8. Represents the KNN classfier.
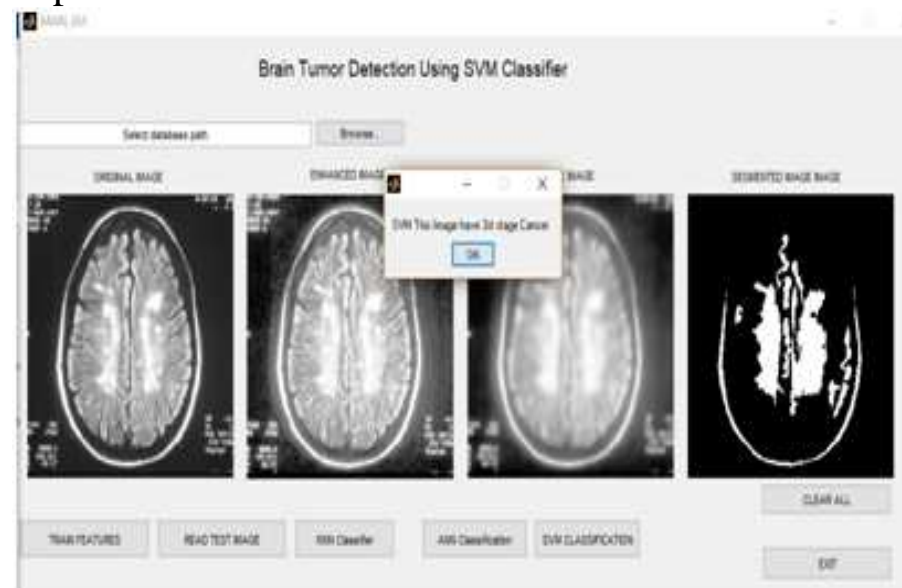

Figure.9. SVM classification
One of the best methods for classifying any image or pattern is SVM. SVM is used to split a set of images into two various classes. The classification is done by finding the hyper-plane that differentiates the two classes very well as given in Fig.9.

The inference drawn from the experimental results shows that SVM proves to be as efficient as the powerful tool ANN in decision making which is proved applying to various images. Sometimes the accuracy rate of using SVM method is little more than ANN.

\begin{tabular}{|c|c|c|c|c|}
\hline MR Image & $\begin{array}{l}\mathrm{K} \\
\mathrm{N} \\
\mathrm{N}\end{array}$ & $\begin{array}{c}\text { Neural } \\
\text { Netwo } \\
\text { rk }\end{array}$ & $\begin{array}{l}\text { S } \\
\text { V } \\
M\end{array}$ & $\begin{array}{c}\text { Groun } \\
\text { d } \\
\text { Truth }\end{array}$ \\
\hline & $\checkmark$ & $\checkmark$ & $\checkmark$ & $\begin{array}{l}\text { This } \\
\text { image } \\
\text { is } \\
\text { havin } \\
\mathrm{g} \\
\text { diseas } \\
\mathrm{e}\end{array}$ \\
\hline & $x$ & $x$ & $\checkmark$ & $\begin{array}{l}\text { This } \\
\text { image } \\
\text { is } \\
\text { havin } \\
\mathrm{g} \\
\text { diseas } \\
\mathrm{e}\end{array}$ \\
\hline & $x$ & $\checkmark$ & $\checkmark$ & $\begin{array}{l}\text { This } \\
\text { image } \\
\text { is } \\
\text { havin } \\
\mathrm{g} \\
\text { diseas } \\
\mathrm{e}\end{array}$ \\
\hline & $\checkmark$ & $x$ & $x$ & $\begin{array}{l}\text { This } \\
\text { image } \\
\text { is not } \\
\text { havin } \\
\mathrm{g} \\
\text { diseas } \\
\mathrm{e}\end{array}$ \\
\hline 0 & $x$ & $x$ & $x$ & $\begin{array}{l}\text { This } \\
\text { image } \\
\text { is not } \\
\text { havin } \\
\mathrm{g} \\
\text { diseas } \\
\mathrm{e}\end{array}$ \\
\hline
\end{tabular}




\section{CONCLUSION}

This method used to detect brain tumor from MR images work accurately for almost many MRI images. The performance of the method adopted uphold the purpose of diagnosis for all types of MRIs. Further advanced kernels or hybrid method associated with support vector machines need to be designed to fit universally for all MRIs. Thus classification efficiency can be improved by adopting efficient hybrid SVMs for large data-sets.

\section{References}

1. Glenn Fung and Olvi L Mangasarian,'Proximal support vector machine classifiers." KDD 2001 San Fransisco CA USA, ACM.

2. G. Kharmega sundararaj, Dr. V. Balamurugan," Robust classification of primary Brain Tumor in Computer Tomography Images using K-NN and Linear SVM.”. 2014 IEEE, pp 1315-1319(IC3I).

3. B. Scholkop, C. J. C. Burges, and A. J. Smola," Advances in kernel methods-support vector learning." MIT press, Cambridge, mass, 1998.

4. Garima singh, Dr. M. A. Ansari,'Efficient Detection of Brain Tumor from MRIs using Kmeans Segmentation and Normalized Histogram." 2016, IEEE.

5. Manugupta, Prof B. V. V. S. N Prabhakar Rao, Dr. Venkateshwaran Rajagopalan, "Brain Tumor Detection in Conventional MR images based on statistical Texture and Morphological features." DOI 10.11091 ICIT.2016.49, IEEE, 2016.

6. Sandhya G, Giribabu Kande, Satya savithri. T," Detection of Normal and abnormal tissues in MR images of the brain using an Advanced Multilevel thresholding technique and kernel SVM classifier.”, 2017, (ICCI-17), IEEE.

7. Gaurav Gupta, Vinay singh.” Brain Tumor segmentation and classification using $\mathrm{Fcm}$ and support vector machine."IRJET 2017, ISSN: 2395-0056, vol-04, issue: 05.

8. Mr. T. Sathies kumar, K. Rashmi, Sreevidhya Ramadoss,"Brain Tumor Detection using SVM classifier." 2017(ICSSS), IEEE, pp318-323.

9. Dr. R. J. Ramteke, Khachane Monali Y, "Automatic Medical Image Classification and Abnormality Detection Using $\mathrm{K}$ Nearest Neighbour", International Journal of Advanced Computer Research (ISSN (print): 2249-7277 ISSN (online): 2277-7970), Volume-2 Number-4 Issue-6 December-2012.

10. Khushboo Singh, SatyaVerma, "Detecting Brain MRI Anomalies By Using SVM Classification", International Journal of Engineering Research and Applications, ISSN: 2248-9622, Vol. 2, Issue 4, June-July 2012.

11. Shweta Jain, "Brain Cancer Classification Using GLCM Based Feature Extraction in Artificial Neural Network", International Journal of Computer Science \& Engineering Technology (IJCSET), ISSN: 2229-3345, Vol. 4 No. 07 Jul 2013.

12. M. Karuna, Ankita Joshi, "Automatic detection and severity analysis of brain tumors using gui in mat lab" IJRET: International Journal of Research in Engineering and Technology, ISSN: 23191163, Volume: 02 Issue: 10, Oct-2013

13. Saptalakar. B. K, Rajeshwari. H, "Segmenttion Based Detection of Brain Tumor,'International Journal of computer and Electronics Research, Vol. 2, pp.20-23, February 2013.

14. Ek Tsoon Tan, James V Miller, Anthony Bianchi, Albert Montello- "Brain Tumor Segmentation With Symmetric Texture And symmetric Intensity Based Decision Forests" GE Global Research, Niskayuna, NY, USA, University of California Riverside, Riverside, CA USA. 